\title{
Thermodynamics of collisional models for Brownian particles: General properties and efficiency
}

\author{
Angel L. L. Stable $\odot$, C. E. Fernández Noa ๑, William G. C. Oropesa $\odot$, and C. E. Fiore $\odot$ \\ Universidade de São Paulo, Instituto de Física, Rua do Matão, 1371, 05508-090 São Paulo, São Paulo, Brasil
}

(Received 1 May 2020; accepted 2 September 2020; published 2 October 2020)

\begin{abstract}
We introduce the idea of collisional models for Brownian particles, in which a particle is sequentially placed in contact with distinct thermal environments and external forces. Thermodynamic properties are exactly obtained, irrespective of the number of reservoirs involved. In the presence of external forces, the entropy production presents a bilinear form in which Onsager coefficients are exactly calculated. Analysis of Brownian engines based on sequential thermal switchings is proposed and considerations about their efficiencies are investigated, taking into account distinct external forces protocols. Our results shed light to an alternative route for obtaining efficient thermal engines based on finite times Brownian machines.
\end{abstract}

DOI: 10.1103/PhysRevResearch.2.043016

\section{INTRODUCTION}

Stochastic thermodynamics has proposed a general and unified scheme for addressing central issues in thermodynamics [1-5]. It includes not only an extension of concepts from equilibrium to nonequilibrium systems but also it deals with the existence of new definitions and bounds [6-9], general considerations about the efficiency of engines at finite time operations [1-3], and others aspects. In all cases, the concept of entropy production $[1,4,10]$ plays a central role, being a quantity continuously produced in nonequilibrium steady states (NESS), whose main properties and features have been extensively studied in the last years, including its usage for typifying phase transitions [11-14].

Basically, a NESS can be generated under two fundamental ways: From fixed thermodynamic forces $[15,16]$ or from time-periodic variation of external parameters [17-20]. In this contribution, we address a different kind of periodic driving, suitable for the description of engineered reservoirs, at which a system interacts sequentially and repeatedly with distinct environments [21-23]. Commonly referred as collisional models, they have been inspired by the assumption that in many cases (e.g., the original Brownian motion) a particle collides only with few molecules of the environment and then the subsequent collision will occur with another fraction of uncorrelated molecules. Collisional models have been viewed as more realistic frameworks in certain cases, encompassing not only particles interacting with a small fraction of the environment but also those presenting distinct drivings over each member of system [24-27] or even species yielding a weak coupling with the reservoir. More recently, they have been (broadly) extended for quantum systems for mimicking the environment, represented by a weak interaction between the

Published by the American Physical Society under the terms of the Creative Commons Attribution 4.0 International license. Further distribution of this work must maintain attribution to the author(s) and the published article's title, journal citation, and DOI. system and a sequential collection of uncorrelated particles [28-30].

With the above in mind, we introduce the concept of repeated interactions for Brownian particles. More specifically, a particle under the influence of a given external force is placed in contact with a reservoir during the time interval and afterwards it is replaced by an entirely different (and independent) set of interactions. Exact expressions for thermodynamic properties are derived and the entropy production presents a bilinear form, in which Onsager coefficients are obtained as function of period. Considerations about the efficiency are undertaken and a suited regime for the system operating as an efficient thermal machine is investigated.

The present study sheds light for fresh perspectives in nonequilibrium thermodynamics, including the possibility of experimental buildings of heat engines based on Brownian dynamics [31-36] with sequential reservoirs. Also, they provide us the extension and validation of recent bounds between currents and entropy production, the so called thermodynamic uncertainty relations (TURs) [8,9,37-41], which has aroused a recent and great interest.

This paper is organized as follows: Secs. II and III present the model description and its exact thermodynamic properties. In Sec. IV we extend analysis for external forces and considerations about efficiency are performed in Sec. V. Conclusions and perspectives are drawn in Sec. VI.

\section{MODEL AND FOKKER-PLANCK EQUATION}

We are dealing with a Brownian particle with mass $m$ sequentially placed in contact with $N$ different thermal reservoirs. Each contact has a duration of $\tau / N$ and occurs during the intervals $\tau_{i-1} \leqslant t<\tau_{i}$, where $\tau_{i}=i \tau / N$ for $i=1, . ., N$, in which the particle evolves in time according to the following Langevin equation:

$$
m \frac{d v_{i}}{d t}=-\alpha_{i} v_{i}+F_{i}(t)+B_{i}(t)
$$

where quantities $v_{i}, \alpha_{i}$, and $F_{i}(t)$ denote the particle velocity, the viscous constant and external force, respectively. From 
now on, we shall express them in terms of reduced quantities: $\gamma_{i}=\alpha_{i} / m$ and $f_{i}(t)=F_{i}(t) / m$. The stochastic force $\zeta_{i}(t)=$ $B_{i}(t) / m$ accounts for the interaction between particle and the $i$ th environment and satisfies the properties

$$
\left\langle\zeta_{i}(t)\right\rangle=0
$$

and

$$
\left\langle\zeta_{i}(t) \zeta_{i^{\prime}}\left(t^{\prime}\right)\right\rangle=2 \gamma_{i} T_{i} \delta_{i i^{\prime}} \delta\left(t-t^{\prime}\right),
$$

respectively, where $T_{i}$ is the bath temperature. Let $P_{i}(v, t)$ be the velocity probability distribution at time $t$, its time evolution is described by the Fokker-Planck (FP) equation $[3,16,42]$

$$
\frac{\partial P_{i}}{\partial t}=-\frac{\partial J_{i}}{\partial v}-f_{i}(t) \frac{\partial P_{i}}{\partial v}
$$

where $J_{i}$ is given by

$$
J_{i}=-\gamma_{i} v P_{i}-\frac{\gamma_{i} k_{\mathrm{B}} T_{i}}{m} \frac{\partial P_{i}}{\partial v} .
$$

It is worth mentioning that above equations are formally identical to description of the overdamped harmonic oscillator subject to the harmonic force $f_{h}=-\bar{k} x$ just by replacing $x \rightarrow v, \bar{k} / \alpha \rightarrow \gamma_{i}, 1 / \alpha \rightarrow \gamma_{i} / m$.

From the FP equation and by performing appropriate partial integrations together boundary conditions in which both $P_{i}(v, t)$ and $J_{i}(v, t)$ vanish at extremities, the time variation of the energy system $U_{i}=\left\langle E_{i}\right\rangle$ in contact with the $i$ th reservoir is given by

$$
\frac{d U_{i}}{d t}=-\frac{m}{2} \int v^{2}\left[\frac{\partial J_{i}}{\partial v}+f_{i}(t) \frac{\partial P_{i}}{\partial v}\right] d v .
$$

The right side of Eq. (6) can be rewritten as $d U_{i} / d t=-\left(\dot{W}_{i}+\right.$ $\dot{Q}_{i}$ ), where $\dot{W}_{i}$ and $\dot{Q}_{i}$ denote the work per unity of time and heat flux from the system to the environment (thermal bath) given by

$$
\dot{W}_{i}=-m\left\langle v_{i}\right\rangle f_{i}(t) \text { and } \dot{Q}_{i}=\gamma_{i}\left(m\left\langle v_{i}^{2}\right\rangle-k_{\mathrm{B}} T_{i}\right),
$$

respectively. In the absence of external forces $\dot{W}_{i}=0$ and all heat flux comes from/goes to the thermal bath.

By assuming the system entropy $S$ is given by $S_{i}(t)=$ $-k_{\mathrm{B}} \int P_{i}(v, t) \ln \left[P_{i}(v, t)\right] d v$ and from the expression for $J_{i}$, one finds that its time derivative is given by

$$
\frac{d S_{i}}{d t}=-k_{\mathrm{B}} \int\left(\frac{J_{i}}{P_{i}}\right)\left(\frac{\partial P_{i}}{\partial v}\right) d v .
$$

As for the mean energy, above expression can be rewritten in the following form:

$$
\frac{d S_{i}}{d t}=\frac{m}{\gamma_{i} T_{i}}\left(\int \frac{J_{i}^{2}}{P_{i}} d v+\gamma_{i} \int v J_{i} d v\right) .
$$

Equation (9) can be interpreted according to the following form: $d S_{i} / d t=\Pi_{i}(t)-\Phi_{i}(t)[16,42]$, where the former term corresponds to the entropy production rate $\Pi_{i}(t)$ and it is strictly positive (as expected). The second term is the the flux of entropy and can also be rewritten more conveniently as

$$
\Phi_{i}(t)=\frac{\dot{Q}_{i}}{T_{i}}=\gamma_{i}\left(\frac{m}{T_{i}}\left\langle v_{i}^{2}\right\rangle-k_{\mathrm{B}}\right) .
$$

If external forces are null and the particle is placed in contact to a single reservoir, then the probability distribution approaches for large times the Gibbs (equilibrium) distribution $P_{i}^{\mathrm{eq}}(v)=e^{-E / k_{\mathrm{B}} T_{i}} / Z$, with $E=m v^{2} / 2$ its kinetic energy and $Z$ the partition function. In such case, $\left\langle v_{i}^{2}\right\rangle=k_{\mathrm{B}} T_{i} / m$ and therefore $\Pi_{\mathrm{eq}}=\Phi_{\mathrm{eq}}=0$ (as expected). Conversely, it will evolve to a nonequilibrium steady state (NESS) when placed in contact with sequential and distinct reservoirs, in which heat is dissipated and the entropy is produced and hence $\Pi_{\mathrm{NESS}}=\Phi_{\mathrm{NESS}}>0$.

\section{EXACT SOLUTION FOR ARBITRARY SET OF SEQUENTIAL RESERVOIRS}

From now on, quantities will be expressed in terms of the "reduced temperature" $\Gamma_{i}=2 \gamma_{i} k_{\mathrm{B}} T_{i} / m$ and $k_{B}=1$. Since we are dealing with a linear force on the velocity, the NESS will also be characterized by a Gaussian probability distribution $P_{i}(v, t)=e^{-\left(v-\left\langle v_{i}\right\rangle\right)^{2} / 2 b_{i}(t)} / \sqrt{2 \pi b_{i}(t)}$ in which both mean $\left\langle v_{i}\right\rangle(t)$ and the variance $b_{i}(t) \equiv\left\langle v_{i}^{2}\right\rangle(t)-\left\langle v_{i}\right\rangle^{2}(t)$ will be in general time-dependent. Their expressions can be calculated from Eqs. (4) and (5) and read

$$
\frac{d}{d t}\left\langle v_{i}\right\rangle=-\gamma_{i}\left\langle v_{i}\right\rangle+f_{i}(t)
$$

and

$$
\frac{d}{d t} b_{i}(t)=-2 \gamma_{i} b_{i}(t)+\Gamma_{i},
$$

respectively, where appropriate partial integrations were performed. Their solutions are given by the following expressions:

$$
\left\langle v_{i}\right\rangle(t)=e^{-\gamma_{i}\left(t-\tau_{i-1}\right)}\left[v_{i-1}^{\prime}+\int_{\tau_{i-1}}^{t} e^{\gamma_{i}\left(t^{\prime}-\tau_{i-1}\right)} f_{i}\left(t^{\prime}\right) d t^{\prime}\right]
$$

and

$$
b_{i}(t)=A_{i-1} e^{-2 \gamma_{i}\left(t-\tau_{i-1}\right)}+\frac{\Gamma_{i}}{2 \gamma_{i}},
$$

respectively, where quantities $v_{i-1}^{\prime} \equiv\left\langle v_{i}\right\rangle\left(\tau_{i-1}\right)$ and $A_{i}$ 's are evaluated by taking into account the set of continuity relations for the averages and variances, $\left\langle v_{i}\right\rangle\left(\tau_{i}\right)=\left\langle v_{i+1}\right\rangle\left(\tau_{i}\right)$ and $b_{i}\left(\tau_{i}\right)=b_{i+1}\left(\tau_{i}\right)$ (for all $\left.i=1, \ldots, N\right)$, respectively. Since the system returns to the initial state after a complete period, $\left\langle v_{1}\right\rangle(0)=\left\langle v_{N}\right\rangle(\tau)$ and $b_{1}(0)=b_{N}(\tau)$, all coefficients can be solely calculated in terms of model parameters, temperature reservoirs and the period. Also, the above conditions state that the probability at each point returns to the same value after every period.

For simplicity, from now on we shall assume the same viscous constant $\gamma_{i}=\gamma$ for all $i$ 's. In the absence of external forces, all $v_{i}^{\prime}$ 's vanish and the entropy production only depends on the coefficients $A_{i}$ 's and $\Gamma_{i}$ 's. Hence, the coefficient $A_{i}$ becomes

$$
A_{i+1}=x A_{i}+\frac{1}{2 \gamma}\left(\Gamma_{i}-\Gamma_{i+1}\right)
$$


where $x=e^{-2 \gamma \tau / N}$ and all of them can be found from a linear recurrence relation

$$
A_{i}=x^{i-1} A_{1}+\frac{1}{2 \gamma} \sum_{l=2}^{i} x^{i-l}\left(\Gamma_{l-1}-\Gamma_{l}\right)
$$

for $i=2, \ldots . N$. As the particle returns to the initial configuration after a complete period, $A_{N}$ then reads

$$
A_{N}=x^{-1} A_{1}+\frac{x^{-1}}{2 \gamma}\left(\Gamma_{1}-\Gamma_{N}\right) .
$$

By equaling Eqs. (16) and (17) for $i=N$, all coefficients $A_{i}$ 's can be finally calculated and are given by

$$
A_{1}=\frac{1}{2 \gamma} \frac{x^{N}}{1-x^{N}} \sum_{l=1}^{N} x^{-l}\left(\Gamma_{l}-\Gamma_{l+1}\right)
$$

and

$$
A_{i}=\frac{1}{2 \gamma} \frac{x^{i-1}}{1-x^{N}}\left[\sum_{l=1}^{i-1} x^{-l}\left(\Gamma_{l}-\Gamma_{l+1}\right)+\sum_{l=i}^{N} x^{N-l}\left(\Gamma_{l}-\Gamma_{l+1}\right)\right],
$$

for $i=1$ and $i>1$, respectively. As we are focusing on the steady-state time-periodic regime, thermodynamic quantities can be averaged over one period $\tau$. The mean entropy production $\bar{\Pi}$ then reads

$$
\bar{\Pi}=\frac{1}{\tau} \sum_{i=1}^{N} \int_{\tau_{i-1}}^{\tau_{i}} \Phi_{i}(t) d t=\frac{\left(1-e^{-2 \gamma \tau / N}\right)}{2 \gamma \tau} \sum_{i=1}^{N} \frac{A_{i}}{\Gamma_{i}} .
$$

From Eqs. (18) and (19), it follows that

$$
\sum_{i=1}^{N} \frac{A_{i}}{\Gamma_{i}}=\frac{x^{N}}{1-x^{N}} \sum_{i, l=1}^{N} x^{-l}\left(\frac{\Gamma_{i+l-1}-\Gamma_{i+l}}{\Gamma_{i}}\right),
$$

and we arrive at an expression for $\bar{\Pi}$ solely dependent on the model parameters

$$
\bar{\Pi}=-\frac{N}{2 \gamma \tau}\left(\frac{1-x}{x}\right)+\frac{1}{2 \gamma \tau} \cdot \frac{x^{N-1}(1-x)^{2}}{1-x^{N}} \sum_{i, l=1}^{N} x^{-l} \frac{\Gamma_{i+l}}{\Gamma_{i}} .
$$

To show that $\bar{\Pi} \geqslant 0$, we resort to the inequality $\sum_{i=1}^{N} \Gamma_{i+l} / \Gamma_{i} \geqslant N \sqrt[N]{\prod_{i=1}^{N} \Gamma_{i+l} / \Gamma_{i}}$ for showing that $\sum_{i=1}^{N} \Gamma_{i+l} / \Gamma_{i} \geqslant N$, and hence Eq. (22) fulfills the condition

$$
\bar{\Pi} \geqslant-\frac{N}{2 \gamma \tau}\left(\frac{1-x}{x}\right)+\frac{N}{2 \gamma \tau}\left(\frac{1-x}{x}\right)=0,
$$

in consistency with the second law of thermodynamics.

As an concrete example, we derive explicit results for the two sequential reservoirs case. From Eqs. (13) and (14), coefficients $A_{1}$ and $A_{2}$ reduce to the following expressions:

$$
A_{1}=\frac{\Gamma_{2}-\Gamma_{1}}{2 \gamma}\left(\frac{1-e^{-\gamma \tau}}{1-e^{-2 \gamma \tau}}\right)=\frac{\Gamma_{2}-\Gamma_{1}}{2 \gamma}\left(\frac{1}{1+e^{\gamma \tau}}\right),
$$

where $A_{2}=-A_{1}$ and hence

$$
\Phi_{1}(t)=\gamma\left(\frac{\Gamma_{2}-\Gamma_{1}}{\Gamma_{1}}\right)\left(\frac{1}{1+e^{2 \gamma \tau}}\right) e^{-2 \gamma t},
$$

for $0 \leqslant t<\tau / 2$ and

$$
\Phi_{2}(t)=\gamma\left(\frac{\Gamma_{1}-\Gamma_{2}}{\Gamma_{2}}\right)\left(\frac{1}{1+e^{2 \gamma \tau}}\right) e^{-2 \gamma\left(t-\frac{\tau}{2}\right)},
$$

$\tau / 2 \leqslant t<\tau$, respectively, whose average entropy production reads

$$
\bar{\Pi}=\left[\frac{\Gamma_{1} \Gamma_{2}}{2 \tau} \tanh \left(\frac{\gamma \tau}{2}\right)\right]\left(\frac{1}{\Gamma_{1}}-\frac{1}{\Gamma_{2}}\right)^{2} .
$$

Note that $\bar{\Pi} \geqslant 0$ and it vanishes when $\Gamma_{1}=\Gamma_{2}$. In the limit of slow ( $\tau \gg 1)$ and fast $(\tau<<1)$ oscillations, $\bar{\Pi}$ approaches to the following asymptotic expressions:

$$
\bar{\Pi} \approx \frac{\Gamma_{1} \Gamma_{2}}{2 \tau}\left(\frac{1}{\Gamma_{1}}-\frac{1}{\Gamma_{2}}\right)^{2} \text { and } \frac{\Gamma_{1} \Gamma_{2} \gamma}{4}\left(\frac{1}{\Gamma_{1}}-\frac{1}{\Gamma_{2}}\right)^{2} \text {, }
$$

respectively, and such a latter expression is independent on the period.

Equation (27) can be conveniently written down as a flux-times-force expression, where the thermodynamic force attempts to the difference of temperatures of reservoirs. Given that the viscous coefficient is the same for all switchings, the thermodynamic force can be more conveniently expressed in terms of difference of $\Gamma_{i}$ 's. More specifically, we have that $\bar{\Pi}=\mathcal{J}_{T} f_{T}$, where $f_{T}=\left(1 / \Gamma_{2}-1 / \Gamma_{1}\right)$ and $\mathcal{J}_{T}$ can also be rewritten as $\mathcal{J}_{T}=L_{T T} f_{T}$, where $L_{T T}$ is the Onsager coefficient given by

$$
L_{T T}=\frac{\Gamma_{1} \Gamma_{2}}{2 \tau} \tanh \left(\frac{\gamma \tau}{2}\right)
$$

Note that $L_{T T} \geqslant 0$ (as expected).

Figure 1 depicts the average entropy production $\bar{\Pi}$ versus $\tau$ for distinct values of $\Gamma_{2}$ and $\Gamma_{1}=1, \gamma=1$. Note that it is monotonically increasing with $f_{T}$ and reproduces above asymptotic limits.

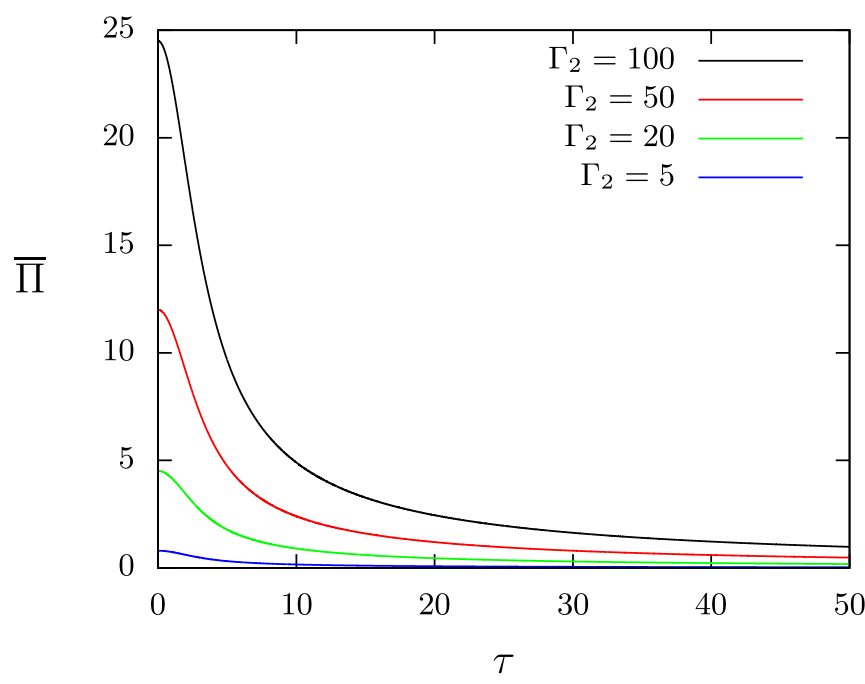

FIG. 1. Mean entropy production $\bar{\Pi}$ versus $\tau$ for distinct temperature sets $\Gamma_{1}=1$ and $\Gamma_{2}$ and $\gamma=1$. 


\section{FORCED BROWNIAN AND SEQUENTIAL RESERVOIRS}

Next, we extend analysis for the case of a Brownian particle in contact with sequential reservoirs and external forces. We shall focus on the two stage case and two simplest external forces protocols: constant and linear drivings. More specifically, the former is given by

$$
f_{i}(t)= \begin{cases}f_{1} ; & 0 \leqslant t<\tau / 2 \\ f_{2} ; & \tau / 2 \leqslant t<\tau\end{cases}
$$

where $f_{1}$ and $f_{2}$ denote their strengths in the first and second half period, respectively, whereas the latter case accounts for forces evolving linearly over the time according to the slopes:

$$
\frac{f_{i}(t)}{\gamma}=\left\{\begin{array}{c}
\lambda_{1} t ; \quad 0 \leqslant t<\tau / 2, \\
\lambda_{2}\left(\frac{\tau}{2}-t\right), \quad \tau / 2 \leqslant t<\tau,
\end{array}\right.
$$

with $\lambda_{1}$ and $\lambda_{2}$ being their amplitudes. It has been considered in Ref. [41] to compare the performance of distinct bounds between currents and the entropy production (TURs). In the presence of external forces, FP equation has the same form of Eq. (14), but now $\left\langle v_{i}\right\rangle(t)$ 's will be different from zero.

\section{A. Constant external forces}

From Eq. (13), the expressions for $\left\langle v_{i}\right\rangle(t)$ 's are given by

$$
\langle v\rangle=\left\{\begin{array}{c}
\left\langle v_{1}\right\rangle(t)=\frac{e^{\gamma \tau / 2}}{\gamma}\left(\frac{f_{2}-f_{1}}{1+e^{\gamma \tau / 2}}\right) e^{-\gamma t}+\frac{f_{1}}{\gamma}, \\
\left\langle v_{2}\right\rangle(t)=\frac{e^{\gamma \tau / 2}}{\gamma}\left(\frac{f_{1}-f_{2}}{1+e^{\gamma \tau / 2}}\right) e^{-\gamma(t-\tau / 2)}+\frac{f_{2}}{\gamma},
\end{array}\right.
$$

for the first or second half of each period, respectively.

The average work and heat per time are given by $\overline{\dot{W}}=$ $\overline{\dot{W}}_{1}+\bar{W}_{2}$ and $\overline{\dot{Q}}=\overline{\dot{Q}}_{1}+\overline{\dot{Q}}_{2}$, respectively, and straightforwardly evaluated from Eq. (7), whose $\bar{W}_{1}$ and $\bar{Q}_{1}$ read

$$
\begin{aligned}
\overline{\dot{W}}_{1} & =-\frac{m f_{1}}{\tau} \int_{0}^{\tau / 2}\left\langle v_{1}\right\rangle d t \\
& =\frac{m f_{1}}{\gamma^{2} \tau}\left(f_{1}-f_{2}\right) \tanh \left(\frac{\gamma \tau}{4}\right)-\frac{m f_{1}^{2}}{2 \gamma}
\end{aligned}
$$

and

$$
\begin{aligned}
\bar{Q}_{1}= & \frac{m}{4 \gamma \tau}\left(\Gamma_{2}-\Gamma_{1}\right) \tanh \left(\frac{\gamma \tau}{2}\right)+\frac{m}{2 \gamma^{2} \tau}\left(f_{1}+f_{2}\right)^{2} \\
& \times \tanh \left(\frac{\gamma \tau}{4}\right)+\frac{2 m f_{1}^{2}}{\gamma^{2} \tau}\left[\frac{\gamma \tau}{4}-\tanh \left(\frac{\gamma \tau}{4}\right)\right],
\end{aligned}
$$

respectively. Analogous expressions are obtained for $\overline{\dot{W}}_{2}$ and $\overline{\dot{Q}}_{2}$ just by exchanging $1 \leftrightarrow 2$. Note that $\overline{\dot{Q}}_{1}+\overline{\dot{Q}}_{2}+\overline{\dot{W}}_{1}+$ $\overline{\dot{W}}_{2}=0$, in consistency with the first law of thermodynamics.

In the same way as before, the steady entropy production per period $\bar{\Pi}$ can be evaluated from Eq. (10) (by taking $k_{\mathrm{B}}=$ 1) and reads

$$
\bar{\Pi}=\frac{2 \gamma}{m}\left(\frac{\overline{\dot{Q}}_{1}}{\Gamma_{1}}+\frac{\overline{\dot{Q}}_{2}}{\Gamma_{2}}\right),
$$

and we arrive at the following expression

$$
\begin{aligned}
\bar{\Pi}= & \frac{1}{2 \tau} \frac{\left(\Gamma_{2}-\Gamma_{1}\right)^{2}}{\Gamma_{1} \Gamma_{2}} \tanh \left(\frac{\gamma \tau}{2}\right)+\frac{1}{\gamma \tau}\left(\frac{1}{\Gamma_{1}}+\frac{1}{\Gamma_{2}}\right) \\
& \times \tanh \left(\frac{\gamma \tau}{4}\right)\left(f_{1}+f_{2}\right)^{2} \\
& +\left(\frac{f_{1}^{2}}{\Gamma_{1}}+\frac{f_{2}^{2}}{\Gamma_{2}}\right)\left[1-\frac{4}{\gamma \tau} \tanh \left(\frac{\gamma \tau}{4}\right)\right] .
\end{aligned}
$$

Since $\gamma \tau \geqslant 0$ and $1-\tanh (x) / x \geqslant 0$, it follows that $\bar{\Pi} \geqslant 0$. Note that $\bar{\Pi}$ reduces to Eq. (27) as $f_{1}=f_{2}=0$.

\section{Bilinear form and Onsager coefficients}

The shape of Eq. (36) is similar to the linear irreversible thermodynamics $[18,19,43]$, in which the entropy production is written down as a sum of flux-times-force expression. This similarity provides to reinterpret Eq. (36) in the following form:

$$
\bar{\Pi}=\mathcal{J}_{T} f_{T}+\mathcal{J}_{1} f_{1}+\mathcal{J}_{2} f_{2},
$$

where forces $f_{T}=\left(1 / \Gamma_{1}-1 / \Gamma_{2}\right)$ and $f_{1(2)}$ have associated fluxes $\mathcal{J}_{T}, \mathcal{J}_{1(2)}$ given by $\mathcal{J}_{T}=L_{T T} f_{T}$ [identical to Eq. (29)],

$$
\mathcal{J}_{1}=L_{11} f_{1}+L_{12} f_{2} \quad \text { and } \quad \mathcal{J}_{2}=L_{21} f_{1}+L_{22} f_{2} \text {, }
$$

respectively, where $L_{11}, L_{12}, L_{21}$, and $L_{22}$ denote their Onsager coefficients given by

$$
L_{11}=\frac{1}{\Gamma_{1}}\left[1-\frac{3}{\gamma \tau} \tanh \left(\frac{\gamma \tau}{4}\right)\right]+\frac{1}{\gamma \tau \Gamma_{2}} \tanh \left(\frac{\gamma \tau}{4}\right)
$$

and

$$
L_{12}=L_{21}=\frac{1}{\gamma \tau}\left(\frac{1}{\Gamma_{1}}+\frac{1}{\Gamma_{2}}\right) \tanh \left(\frac{\gamma \tau}{4}\right),
$$

respectively. Coefficients $L_{22}$ and $L_{21}$ have the same shape of $L_{11}$ and $L_{12}$ by replacing $1 \leftrightarrow 2$, respectively. Besides, $L_{11}$ and $L_{22} \geqslant 0$ (as should be) and they satisfy the inequality $4 L_{11} L_{22}-\left(L_{12}+L_{21}\right)^{2} \geqslant 0$, in consistency with the positivity of the entropy production.

\section{B. Time-dependent external forces}

By repeating the previous calculations for linear external forces the mean velocities $\left\langle v_{i}\right\rangle(t)$ 's are given by

$$
\langle v\rangle=\left\{\begin{array}{l}
\left\langle v_{1}\right\rangle(t)=\frac{1}{\gamma}\left\{\lambda_{1}(\gamma t-1)\right. \\
\left.+e^{-\gamma t}\left[\lambda_{1}+\left(\lambda_{2} e^{\frac{\gamma \tau}{2}}-\lambda_{1}\right) \alpha(\gamma, \tau)\right]\right\}, \\
\left\langle v_{2}\right\rangle(t)=\frac{1}{\gamma}\left\{-\lambda_{2}\left[\gamma\left(t-\frac{\tau}{2}\right)-1\right]\right. \\
\left.+e^{-\gamma\left(t-\frac{\tau}{2}\right)}\left[\left(\lambda_{1} e^{\frac{\gamma \tau}{2}}-\lambda_{2}\right) \alpha(\gamma, \tau)-\lambda_{2}\right]\right\},
\end{array}\right.
$$

where

$$
\alpha(\gamma, \tau)=\frac{2-e^{\frac{\gamma \tau}{2}}(\gamma \tau-2)}{2\left(e^{\gamma \tau}-1\right)},
$$

respectively. Although more complex than the previous case, the mean work and heat per time are evaluated analogously 

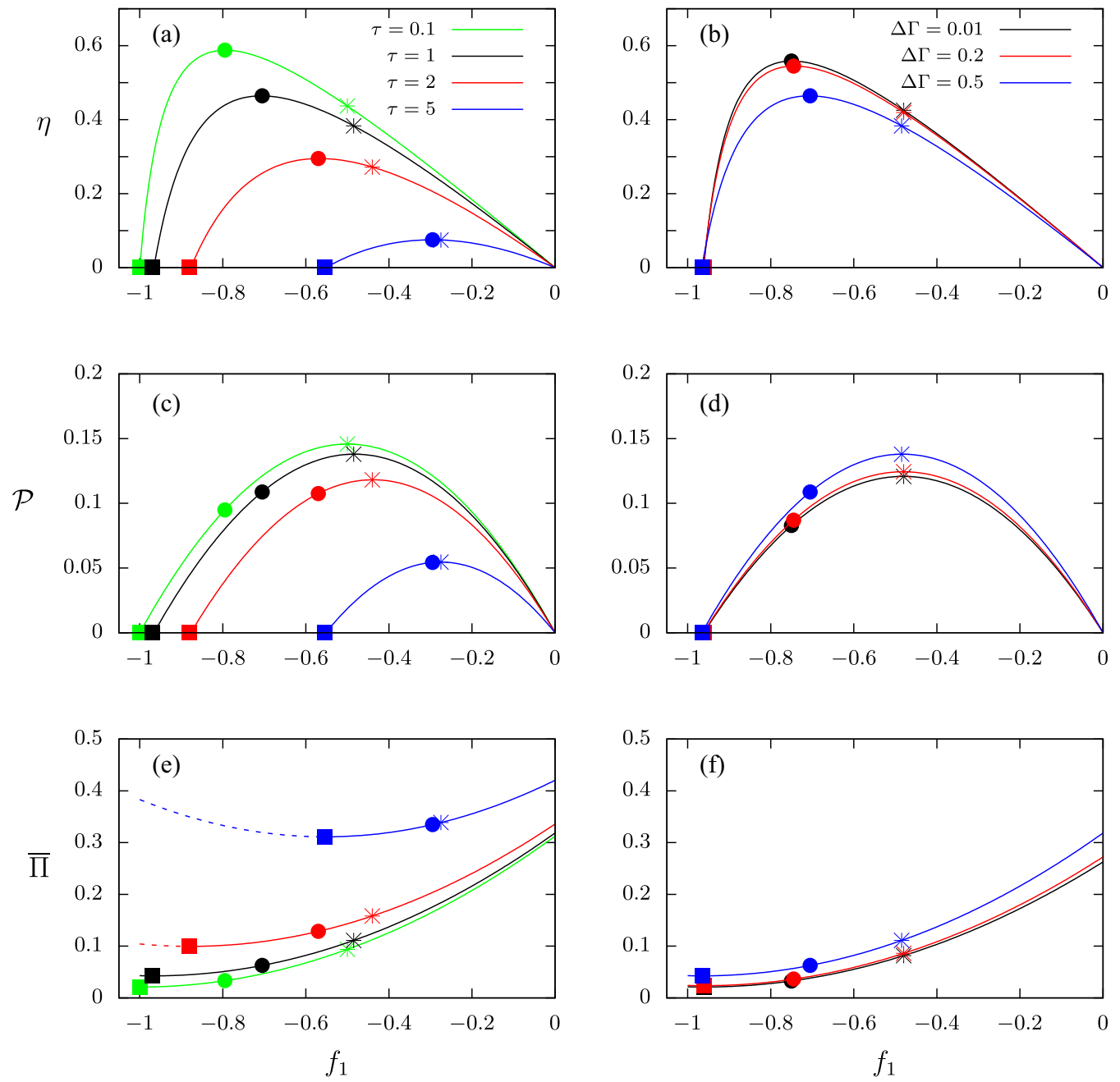

FIG. 2. Panels (a) and (b) depict the efficiency $\eta$ versus $f_{1}$ for distinct periods $\tau$ (for $\Delta \Gamma=0.5$ ) and $\Delta \Gamma$ 's (for $\tau=1$ ), respectively. In both cases, $\Gamma_{1}=2$ and $f_{2}=1$. Symbols $\bullet$, "stars," and "squares" denote the $f_{1 m E}, f_{1 m P}$, and $f_{1 m s}$, respectively. Panels (c) and (d) show the corresponding power $\mathcal{P}$, whereas (e) and (f) the average entropy production rate $\bar{\Pi}$. Dashed lines show the values of $f_{1}$ the system can not be operated as a thermal machine.

from expressions for $\left\langle v_{i}\right\rangle(t)$ 's and $b_{i}(t)$ 's, whose values averaged over a cycle read

$$
\begin{aligned}
\overline{\dot{W}}= & -\bar{Q}=-\mathcal{A}\left\{e^{\gamma \tau} \varphi_{+}(\gamma, \tau, \xi)\right. \\
& \left.+12 e^{\frac{\gamma \tau}{2}}\left(\gamma^{2} \tau^{2} \xi-4\right)+\varphi_{-}(\gamma, \tau, \xi)\right\},
\end{aligned}
$$

where parameters $\mathcal{A}, \xi$ and $\varphi_{ \pm}(\gamma, \tau, \xi)$ read

$$
\mathcal{A}=\frac{m\left(\lambda_{1}+\lambda_{2}\right)^{2}}{24 \gamma^{2} \tau\left(e^{\gamma \tau}-1\right)}, \quad \xi=\frac{\lambda_{1} \lambda_{2}}{\left(\lambda_{1}+\lambda_{2}\right)^{2}},
$$

and

$$
\varphi_{ \pm}(\gamma, \tau, \xi)=\gamma^{2} \tau^{2}(2 \xi-1)(3 \pm \gamma \tau)+24(1 \pm \gamma \tau \xi),
$$

respectively.

\section{Bilinear form and Onsager coefficients}

As in the previous case, the entropy production has also the shape of Eqs. (37) and (38) given by $\bar{\Pi}=\mathcal{J}_{T} f_{T}+\mathcal{J}_{1} \lambda_{1}+$ $\mathcal{J}_{2} \lambda_{2}$, being $L_{T T}$ the same to Eq. (29), whereas the other
Onsager coefficients read

$$
\begin{aligned}
L_{11}= & \frac{1}{\Gamma_{1}}\left[\frac{\gamma^{2} \tau^{2}}{12}-\frac{\gamma \tau\left(2 e^{\gamma \tau}+1\right)}{4\left(e^{\gamma \tau}-1\right)}+\frac{1}{1+e^{-\frac{\gamma \tau}{2}}}\right. \\
& \left.+\frac{1}{\gamma \tau} \tanh \left(\frac{\gamma \tau}{4}\right)\right]+\frac{1}{\Gamma_{2}} \frac{\left[e^{\frac{\gamma \tau}{2}}(\gamma \tau-2)+2\right]^{2}}{4 \gamma \tau\left(e^{\gamma \tau}-1\right)},
\end{aligned}
$$

and

$$
L_{12}=\frac{\left(2 e^{\frac{\gamma \tau}{2}}-\gamma \tau-2\right)\left(2 e^{\frac{\gamma \tau}{2}}-\gamma \tau e^{\frac{\gamma \tau}{2}}-2\right)\left(\Gamma_{1}+\Gamma_{2}\right)}{4 \gamma \tau\left(e^{\gamma \tau}-1\right) \Gamma_{1} \Gamma_{2}},
$$

respectively. Coefficients $L_{22}$ and $L_{21}$ are again identical to $L_{11}$ and $L_{12}$ by exchanging $1 \leftrightarrow 2$. Also, it is straightforward to verify that $L_{11}$ and $L_{22}$ are strictly positive and $4 L_{11} L_{22}-$ $\left(L_{12}+L_{21}\right)^{2} \geqslant 0$.

\section{EFFICIENCY}

Distinct works have tackled the conditions in which periodically driven systems can operate as thermal machines 

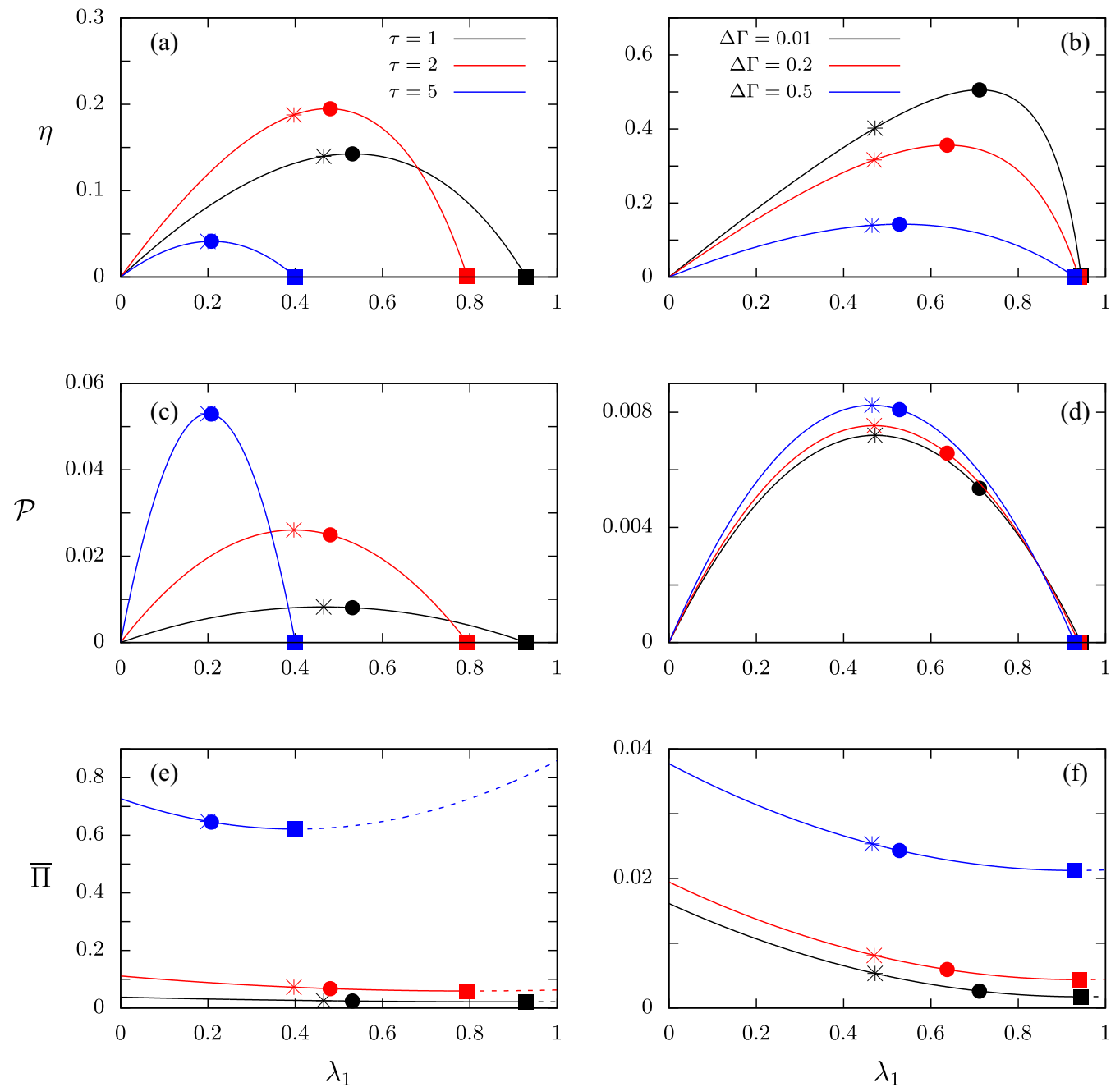

FIG. 3. Panels (a) and (b) depict the efficiency $\eta$ versus $\lambda_{1}$ for distinct periods $\tau$ (for $\Delta \Gamma=0.5$ ) and $\Delta \Gamma$ 's (for $\tau=1$ ), respectively. In both cases, $\Gamma_{1}=2$ and $\lambda_{2}=1$. Symbols $\bullet$, "stars," and "squares" denote the $\lambda_{1 m E}, \lambda_{1 m P}$, and $\lambda_{1 m s}$, respectively. Panels (c) and (d) show the corresponding power $\mathcal{P}$, whereas (e) and (f) the average entropy production rate $\bar{\Pi}$. Dashed lines show the values of $\lambda_{1}$ the system can not be operated as a thermal machine.

[18,43-47]. The conversion of a given type of energy into another one requires the existence of a generic force $X_{1}$ operating against its flux $J_{1} X_{1} \leqslant 0$ counterbalancing with driving forces $X_{2}$ and $X_{T}$ in which $J_{2} X_{2}+J_{T} X_{T} \geqslant 0$. A measure of efficiency $\eta$ is given by

$$
\begin{aligned}
\eta & =-\frac{\mathcal{J}_{1} X_{1}}{\mathcal{J}_{2} X_{2}+\mathcal{J}_{T} X_{T}} \\
& =-\frac{L_{11} X_{1}^{2}+L_{12} X_{1} X_{2}}{L_{21} X_{2} X_{1}+L_{22} X_{2}^{2}+L_{T T} X_{T}^{2}},
\end{aligned}
$$

where in such case $X_{T}=f_{T}$ and we have taken into account Eq. (37) for relating fluxes and Onsager coefficients. Taking into account that the best machine aims at maximizing the efficiency and minimizing the dissipation $\bar{\Pi}$ for a given power output $\mathcal{P}=-\Gamma_{1} \mathcal{J}_{1} X_{1}$, it is important to analyze the role of three load forces, $X_{1 m P}, X_{1 m E}$, and $X_{1 m s}$, in which the power output and efficiency are maximum and the dissipation is minimum, respectively [18]. Their values can be obtained straightforwardly from expressions for $\mathcal{P}$ and Eq. (45), respectively. Due to the present symmetric relation between Onsager coefficients $L_{12}=L_{21}$ (in both cases), they acquire simpler forms and read $2 X_{1 m P}=-L_{12} X_{2} / L_{11}$,

$$
X_{1 m E}=\frac{1}{L_{11} L_{12} X_{2}}\left[-L_{11}\left(L_{22} X_{2}^{2}+L_{T T} X_{T}^{2}\right)+A\left(X_{2}, X_{T}\right)\right]
$$

with $A\left(X_{2}, X_{T}\right)$ being given by

$$
\begin{aligned}
A\left(X_{2}, X_{T}\right)= & \sqrt{L_{11}\left(L_{22} X_{2}^{2}+L_{T T} X_{T}^{2}\right)} \\
& \times \sqrt{\left[L_{11}\left(L_{22} X_{2}^{2}+L_{T T} X_{T}^{2}\right)-L_{12}^{2} X_{2}^{2}\right]},
\end{aligned}
$$

and $X_{1 m S}=-L_{12} X_{2} / L_{11}=2 X_{1 m P}$, respectively, where $X_{i}=$ $f_{i}$ and $\lambda_{i}$ for the constant and linear drivings, respectively. The efficiencies at minimum dissipation, maximum power and its maximum value become $\eta_{m s}=0$,

$$
\eta_{m P}=\frac{L_{12}^{2} X_{2}^{2}}{2\left(2 L_{22} L_{11}-L_{12}^{2}\right) X_{2}^{2}+4 L_{T T} L_{11} X_{T T}^{2}},
$$


and

$\eta_{m E}=\frac{1}{L_{12}^{2} X_{2}^{2}}\left[2 L_{11}\left(L_{22} X_{2}^{2}+L_{T T} X_{T T}^{2}\right)-L_{12}^{2} X_{2}^{2}-2 A\left(X_{2}, X_{T}\right)\right]$,

respectively, and finally their associated power outputs read $\mathcal{P}_{m S}=0, \mathcal{P}_{m P}=\Gamma_{1} L_{12}^{2} X_{2}^{2} / 4 L_{11}$, and

$$
\begin{aligned}
\mathcal{P}_{m E}= & \frac{\Gamma_{1}}{L_{11} L_{12}^{2} X_{2}^{2}} \\
& \times\left[L_{11}\left(L_{22} X_{2}^{2}+L_{T T} X_{T}^{2}\right)-A\left(X_{2}, X_{T}\right)-L_{12}^{2} X_{2}^{2}\right] \\
& \times\left[L_{11}\left(L_{22} X_{2}^{2}+L_{T T} X_{T}^{2}\right)-A\left(X_{2}, X_{T}\right)\right]
\end{aligned}
$$

respectively. We pause to make a few comments: First, above expressions extend the findings from Ref. [18] for a couple of driving forces. Second, both efficiency and power vanish when $X_{1}=X_{1 m s}$ and $X_{1}=0$ and are strictly positive between those limits. Hence the physical regime in which the system can operate as an engine is bounded by the lowest entropy production $\bar{\Pi}_{m S}=L_{T T} X_{T}^{2}+\left(L_{22}-L_{12}^{2} / L_{11}\right) X_{2}^{2}$ and the value $\bar{\Pi}^{*}=L_{T T} X_{T}^{2}+L_{22} X_{2}^{2}$. Third, despite the long expressions for Eqs. (49) and (50), powers $\mathcal{P}_{m P}, \mathcal{P}_{m E}$ and efficiencies $\eta_{m P}, \eta_{m E}$ are linked through a couple of simple expressions (in similarity with Refs. [18,46]):

$$
\eta_{m P}=\frac{\eta_{m E}}{1+\eta_{m E}^{2}} \quad \text { and } \quad \frac{\mathcal{P}_{m E}}{\mathcal{P}_{m P}}=1-\eta_{m E}^{2},
$$

and they imply that $0 \leqslant \eta_{m P}<\eta_{m E}$ (with $0 \leqslant \eta_{m E} \leqslant 1$ and $0 \leqslant \eta_{m P} \leqslant 1 / 2$ ) and $0 \leqslant \mathcal{P}_{m E} \leqslant \mathcal{P}_{m P}$. Fourth and last, the achievement of most efficient machine $\eta_{m E}=1$ implies that the system has to be operated at null power $\mathcal{P}_{m E}=0$ and hence the projection of a machine operating for finite $\mathcal{P}_{m P} / \mathcal{P}_{m E}$ will imply at a loss of its efficiency.

Our purpose here aims at not only extending relevant concepts about efficiency for Brownian particles in contact with sequential reservoirs, but also to show that a desired compromise between maximum power and maximum efficiency can be achieved by adjusting conveniently the model parameters (such as the period and the driving). From expressions for Onsager coefficients, aforementioned quantities are evaluated, as depicted in Figs. 2 and 3 for distinct periods $\tau$ and temperature differences $\Delta \Gamma$ 's for constant and linear drivings, respectively. In both cases, quantities follow theoretical predictions and exhibit similar portraits, in which efficiencies and power outputs present maximum values at $f_{1 m E}\left(\lambda_{1 m E}\right)$ and $f_{1 m P}\left(\lambda_{1 m P}\right)$, respectively. The loss of efficiency from the maximum $\eta_{m E}$ as $f_{1}\left(\lambda_{1}\right)$ goes up (down) is signed by the increase of dissipation (as expected) until vanishing when $\bar{\Pi}=\bar{\Pi}^{*}$. For the constant driving, absolute values of forces and efficiencies increase as the period $\tau$ [see, e.g., panels (a)] and/or temperature differences [see, e.g., panels (b)] are lowered. In such a case, $\Gamma_{1} \approx \Gamma_{2}=\Gamma, \Delta \Gamma=$ $\Gamma_{1}-\Gamma_{2}<<1$ and the thermodynamic force $f_{T}$ approaches to $f_{T} \approx \Delta \Gamma / \Gamma^{2}$. Onsager coefficients become simpler in the limit of fast switchings, $\tau \rightarrow 0$ and $L_{11}, L_{22}, L_{12}$ approach to $\left(\Gamma_{1}+\Gamma_{2}\right) /\left(4 \Gamma_{1} \Gamma_{2}\right)$. Some remarkable quantities then approach to the asymptotic values $f_{1 m s} \rightarrow-f_{2}=2 f_{1 m P}$ and

$$
\eta_{m P} \rightarrow \frac{f_{2}^{2}\left(\Gamma_{1}+\Gamma_{2}\right)}{2\left[f_{2}^{2}\left(\Gamma_{1}+\Gamma_{2}\right)+2 \Delta \Gamma^{2}\right]},
$$

respectively. For $\Gamma_{1} \approx \Gamma_{2}, \eta_{m P} \rightarrow 1 / 2, \eta_{m E} \rightarrow 1$, and $\mathcal{P}_{m P}$ reads $\mathcal{P}_{m P} \rightarrow f_{2}^{2} / 8$ and thereby the limit of an ideal machine is achieved for low periods and equal temperatures. Similar features are verified for the linear driving, including increasing efficiencies as both $\tau$ and $\Delta \Gamma$ decreases. However, they are marked by a reentrant behavior for $\tau<<1$ and $\Delta \Gamma \neq 0$ [see, e.g., Figs. 3(a) and 5]. It moves for lower $\tau$ 's as $\Delta \Gamma$ goes down and the limit of ideal machine, $\eta_{m P} \rightarrow 1 / 2$ and $\eta_{m E} \rightarrow 1$, is also recovered when both $\tau \rightarrow 0$ for $\Delta \Gamma \rightarrow 0$.
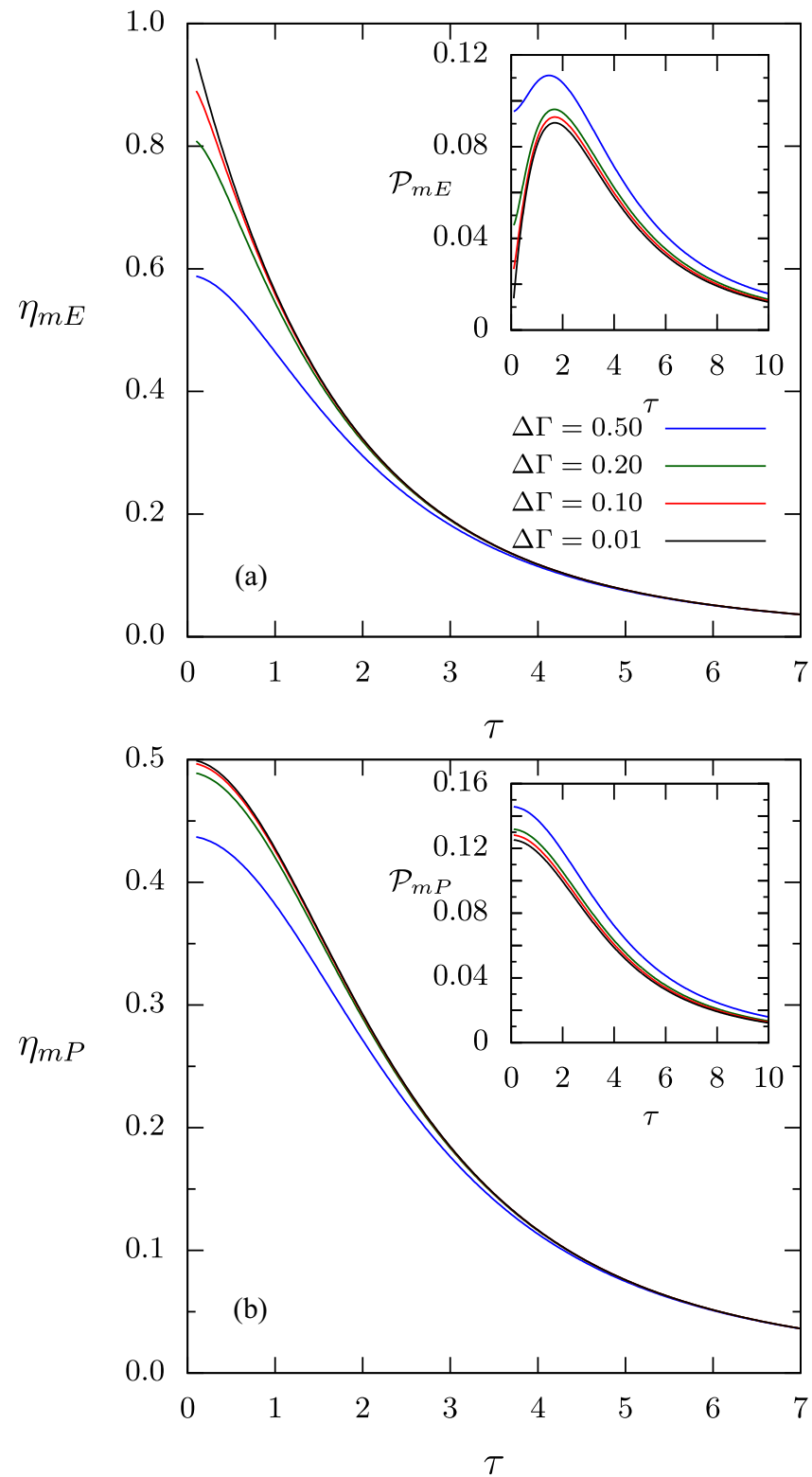

FIG. 4. For $\Gamma_{1}=2, f_{2}=1$ and distinct $\Delta \Gamma$ 's, the comparison between maximum efficiency [panel (a)] and efficiency at maximum power [panel (b)] for constant drivings. Insets: The corresponding power outputs $\mathcal{P}$ 's versus $\tau$. 

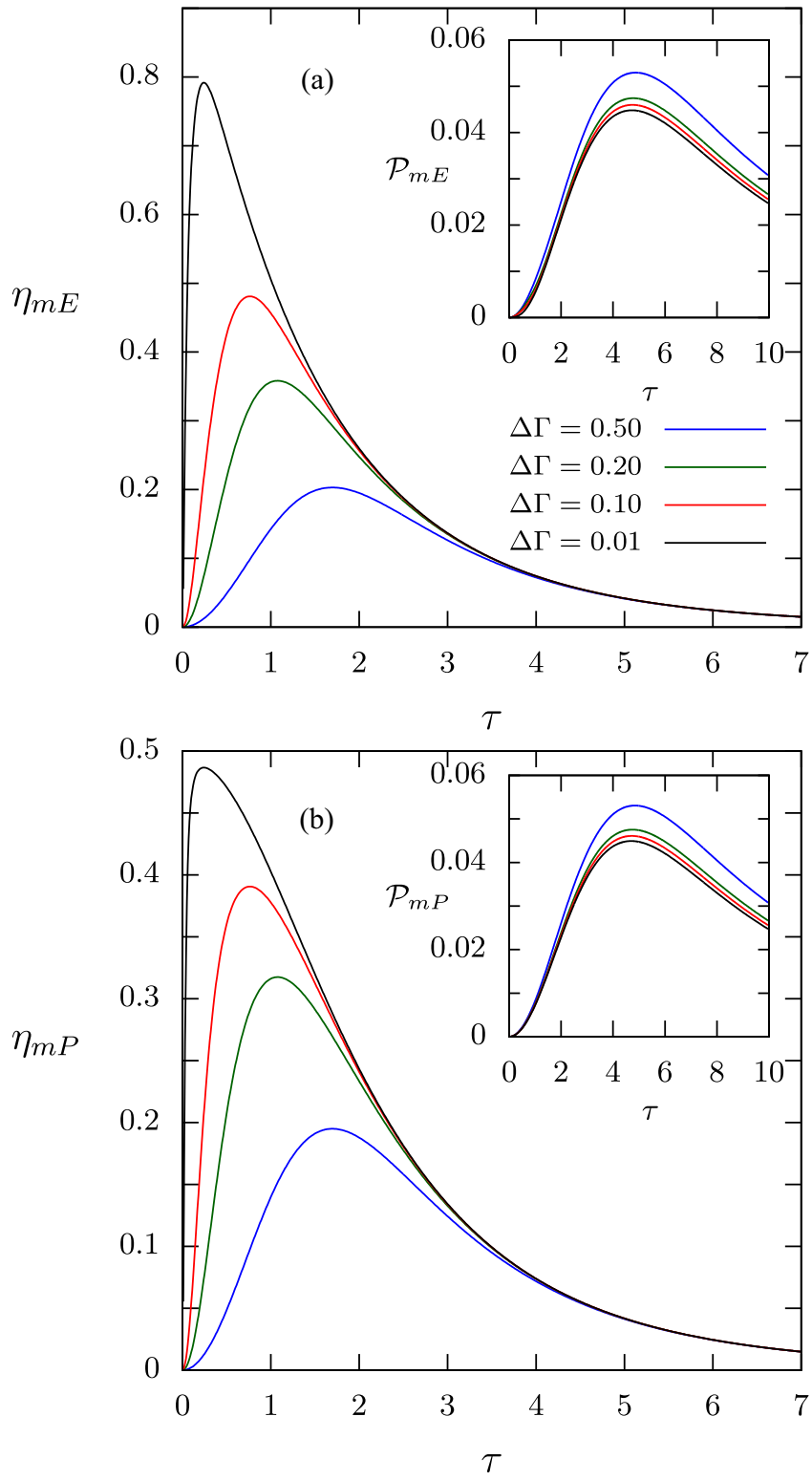

FIG. 5. For $\Gamma_{1}=2, \lambda_{2}=1$, and distinct $\Delta \Gamma$ 's, the comparison between maximum efficiency [panel (a)] and efficiency at maximum power [panel (b)] for linear drivings. Insets: The corresponding power outputs $\mathcal{P}$ 's versus $\tau$.
Other differences between protocols are appraised in Figs. 4 and 5. For finite difference of temperatures, the constant driving is always more efficient than the linear one and their power outputs are also superior. The maximum efficiency curves (linear drivings) are also reentrant, whose maxima values increase and deviate for lower $\tau$ 's as $\Delta \Gamma$ decreases.

We close this section by remarking that although short periods indicates a general route for optimizing the efficiency of thermal machines in contact to sequential reservoirs, the present description provides to properly tune the period and forces to obtain the desirable compromise between maximum efficiency and power.

\section{CONCLUSIONS}

The thermodynamics of a Brownian particle periodically placed in contact with sequential thermal reservoirs is introduced. We have obtained explicit (exact) expressions for relevant quantities, such as heat, work, and entropy production. Generalization for an arbitrary number of sequential reservoirs and the influence of external forces were considered. Considerations about the efficiency were undertaken, in which Brownian machines can be properly operated ensuring the reliable compromise between efficiency and power for small switching periods.

As a final comment, we mention the several perspectives to be addressed. First, it might be very interesting to extend such a study for other external forces protocols (e.g., sinusoidal time-dependent ones) as well as for time asymmetric switchings, to compare their efficiencies, mainly with the linear driving case. Finally, it would be remarkable to verify the validity of recent proposed uncertainties relations (TURs) for Fokker-Planck equations [39,41], in such a class of systems.

\section{ACKNOWLEDGMENTS}

We acknowledge Karel Proesmans and Mário J. de Oliveira for careful readings of the manuscript and useful suggestions. C.E.F. acknowledges the financial support from Fundação de Amparo à Pesquisa do estado de São Paulo (FAPESP) under Grant No. 2018/02405-1. Authors also acknowledge the financial support from Brazilian Agencies CAPES and CNPq.
[1] I. Prigogine, Introduction to Thermodynamics of Irreversible Processes (Interscience, New York, 1965).

[2] S. R. De Groot and P. Mazur, Non-Equilibrium Thermodynamics (North-Holland, Amsterdam, 1962).

[3] T. Tomé and M. J. De Oliveira, Stochastic Dynamics and Irreversibility (Springer, Berlin, 2015).

[4] U. Seifert, Rep. Prog. Phys. 75, 126001 (2012).

[5] C. Van den Broeck and M. Esposito, Physica A 418, 6 (2015).

[6] C. Jarzynski, Phys. Rev. Lett. 78, 2690 (1997).

[7] O.-P. Saira, Y. Yoon, T. Tanttu, M. Möttönen, D. V. Averin, and J. P. Pekola, Phys. Rev. Lett. 109, 180601 (2012).

[8] K. Proesmans and C. Van den Broeck, Europhys. Lett. 119, 20001 (2017).
[9] A. C. Barato and U. Seifert, Phys. Rev. Lett. 114, 158101 (2015).

[10] J. Schnakenberg, Rev. Mod. Phys. 48, 571 (1976).

[11] C. E. Fernández Noa, P. E. Harunari, M. J. de Oliveira, and C. E. Fiore, Phys. Rev. E 100, 012104 (2019).

[12] T. Herpich, J. Thingna, and M. Esposito, Phys. Rev. X 8, 031056 (2018).

[13] T. Herpich and M. Esposito, Phys. Rev. E 99, 022135 (2019).

[14] B. O. Goes, C. E. Fiore, and G. T. Landi, Phys. Rev. Res. 2, 013136 (2020).

[15] H. Ge, M. Qian, and H. Qian, Phys. Rep. 510, 87 (2012).

[16] T. Tomé and M. J. de Oliveira, Phys. Rev. E 91, 042140 (2015). 
[17] K. Brandner, K. Saito, and U. Seifert, Phys. Rev. X 5, 031019 (2015).

[18] K. Proesmans, B. Cleuren, and C. Van den Broeck, Phys. Rev. Lett. 116, 220601 (2016).

[19] K. Proesmans and C. E. Fiore, Phys. Rev. E 100, 022141 (2019).

[20] B. Cleuren and K. Proesmans, Physica A 552, 122789 (2020).

[21] M. Pezzutto, M. Paternostro, and Y. Omar, New J. Phys. 18, 123018 (2016).

[22] M. Pezzutto, M. Paternostro, and Y. Omar, Quant. Sci. Technol. 4, 025002 (2019).

[23] G. T. Landi, E. Novais, M. J. de Oliveira, and D. Karevski, Phys. Rev. E 90, 042142 (2014).

[24] C. H. Bennett, Int. J. Theor. Phys. 21, 905 (1982).

[25] K. Maruyama, F. Nori, and V. Vedral, Rev. Mod. Phys. 81, 1 (2009).

[26] T. Sagawa, J. Stat. Mech.: Theory Exp. (2014) P03025.

[27] J. M. Parrondo, J. M. Horowitz, and T. Sagawa, Nat. Phys. 11, 131 (2015).

[28] V. Giovannetti and G. M. Palma, Phys. Rev. Lett. 108, 040401 (2012).

[29] P. Strasberg, G. Schaller, T. Brandes, and M. Esposito, Phys. Rev. X 7, 021003 (2017).

[30] F. L. S. Rodrigues, G. De Chiara, M. Paternostro, and G. T. Landi, Phys. Rev. Lett. 123, 140601 (2019).

[31] C. Van den Broeck, Phys. Rev. Lett. 95, 190602 (2005).
[32] T. Schmiedl and U. Seifert, Europhys. Lett. 83, 30005 (2008).

[33] F. Berger, T. Schmiedl, and U. Seifert, Phys. Rev. E 79, 031118 (2009).

[34] A. Dechant, N. Kiesel, and E. Lutz, Europhys. Lett. 119, 50003 (2017).

[35] Y. Izumida and K. Okuda, Eur. Phys. J. B 77, 499 (2010).

[36] D. Abreu and U. Seifert, Europhys. Lett. 94, 10001 (2011).

[37] A. C. Barato and U. Seifert, J. Phys. Chem. B 119, 6555 (2015).

[38] T. R. Gingrich, J. M. Horowitz, N. Perunov, and J. L. England, Phys. Rev. Lett. 116, 120601 (2016).

[39] Y. Hasegawa and T. Van Vu, Phys. Rev. E 99, 062126 (2019).

[40] T. Van Vu and Y. Hasegawa, Phys. Rev. E 100, 012134 (2019).

[41] T. Van Vu and Y. Hasegawa, Phys. Rev. Res. 2, 013060 (2020).

[42] T. Tomé and M. J. de Oliveira, Phys. Rev. E 82, 021120 (2010).

[43] K. Proesmans and C. Van den Broeck, Phys. Rev. Lett. 115, 090601 (2015).

[44] M. Bauer, K. Brandner, and U. Seifert, Phys. Rev. E 93, 042112 (2016).

[45] A. Rosas, C. Van den Broeck, and K. Lindenberg, Phys. Rev. E 94, 052129 (2016).

[46] K. Proesmans and C. Van den Broeck, Chaos: Interdiscip. J Nonlin. Sci. 27, 104601 (2017).

[47] A. Rosas, C. Van den Broeck, and K. Lindenberg, Phys. Rev. E 96, 052135 (2017). 